\title{
Construction of a Biomimetic Surface on Microfluidic Chips For Biofouling Resistance
}

\author{
Hongyan Bi, ${ }^{1 \#}$ Wei Zhong, ${ }^{2 \#}$ Sheng Meng, ${ }^{2}$ Jilie Kong, ${ }^{1}$ Pengyuan Yang, ${ }^{1}$ and Baohong Liu ${ }^{1 *}$ \\ ${ }^{1}$ Department of Chemistry, and ${ }^{2}$ Department of Macromolecular Science, Fudan University, \\ Shanghai 200433, People's Republic of China
}

\section{Supporting Information}

SI-Figure 1 Confocal fluorescence images of (a) native PMMA and (b) PMB modified substrates with adsorption of $2 \mathrm{mg} / \mathrm{mL}$ BSA-FITC.

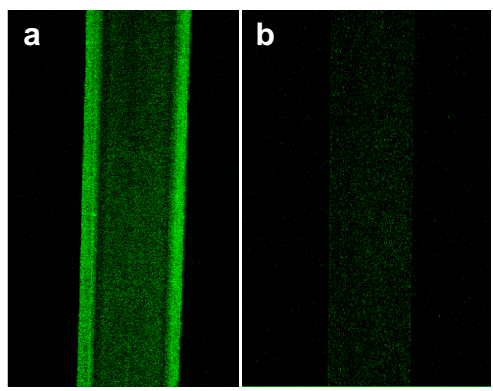


SI-Figure 2 The nitrogen content on the surface of native and PMB modified PMMA substrate for protein adsorption in healthy human serum samples, which was calculated from XPS results (* The value was subtracted by $\mathrm{N}$ content in phosphocholine groups on PMB-coated layer).

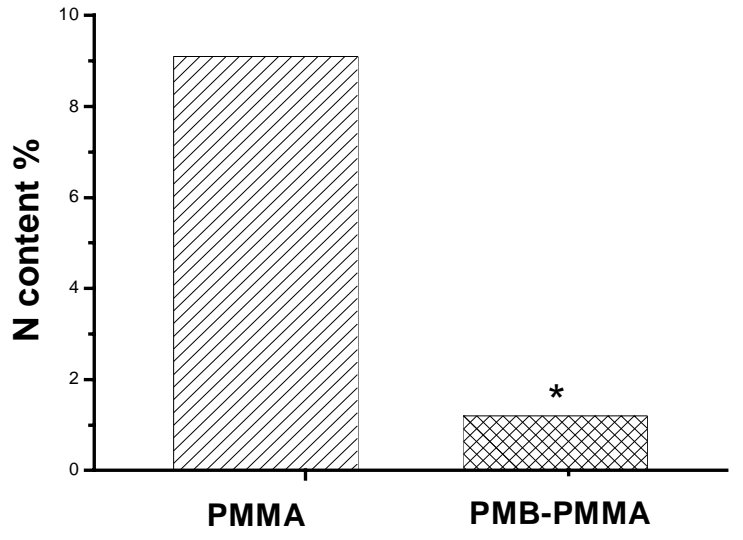


SI-Figure 3 Electropherograms of separation of $0.35 \mu \mathrm{M}$ FITC-Lysozyme and $0.19 \mu \mathrm{M}$ FITC-BSA on the blank PMMA chip. Note the time starting to sample injection is labeled in the chart. Condition: Field strength for separation, $250 \mathrm{~V} / \mathrm{cm}$. The first peak corresponded to FITC-Lysozyme, and the second one to FITC-BSA.

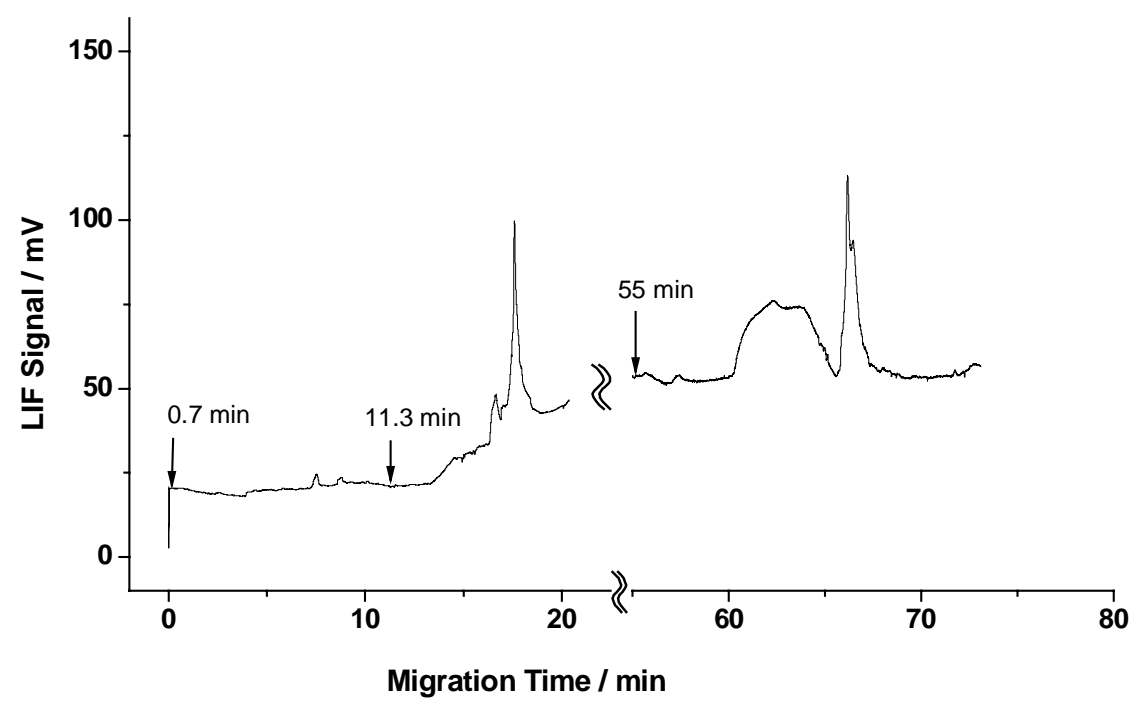

